OPEN ACCESS

Edited by:

Peter Sörös,

University of Oldenburg, Germany

Reviewed by:

Domenico Zaca,

Siemens, Italy

Andrei Holodny,

Cornell University, United States

*Correspondence:

Joshua S. Shimony

shimonyj@wustl.edu

Specialty section:

This article was submitted to Applied Neuroimaging, a section of the journal Frontiers in Neurology

Received: 02 March 2020

Accepted: 30 June 2020

Published: 05 August 2020

Citation:

Luckett P, Lee JJ, Park KY, Dierker D, Daniel AGS, Seitzman BA, Hacker $C D$, Ances BM, Leuthardt EC, Snyder AZ and Shimony JS (2020) Mapping of the Language Network With Deep Learning. Front. Neurol. 11:819. doi: 10.3389/fneur.2020.00819

\section{Mapping of the Language Network With Deep Learning}

\author{
Patrick Luckett ${ }^{1}$, John J. Lee ${ }^{2}$, Ki Yun Park ${ }^{2}$, Donna Dierker ${ }^{2}$, Andy G. S. Daniel ${ }^{3}$, \\ Benjamin A. Seitzman ${ }^{1}$, Carl D. Hacker ${ }^{4}$, Beau M. Ances ${ }^{1}$, Eric C. Leuthardt ${ }^{3,4}$, \\ Abraham Z. Snyder ${ }^{1,2}$ and Joshua S. Shimony ${ }^{2 *}$ \\ ${ }^{1}$ Department of Neurology, Washington University School of Medicine, St. Louis, MO, United States, ${ }^{2}$ Mallinckrodt Institute \\ of Radiology, Washington University School of Medicine, St. Louis, MO, United States, ${ }^{3}$ Department of Biomedical \\ Engineering, Washington University, St. Louis, MO, United States, ${ }^{4}$ Department of Neurosurgery, Washington University \\ School of Medicine, St. Louis, MO, United States
}

Background: Pre-surgical functional localization of eloquent cortex with task-based functional MRI (T-fMRI) is part of the current standard of care prior to resection of brain tumors. Resting state fMRI (RS-fMRI) is an alternative method currently under investigation. Here, we compare group level language localization using T-fMRI vs. RS-fMRI analyzed with 3D deep convolutional neural networks (3DCNN).

Methods: We analyzed data obtained in 35 patients with brain tumors that had both language T-fMRI and RS-MRI scans during pre-surgical evaluation. The T-fMRI data were analyzed using conventional techniques. The language associated resting state network was mapped using a 3DCNN previously trained with data acquired in $>2,700$ normal subjects. Group level results obtained by both methods were evaluated using receiver operator characteristic analysis of probability maps of language associated regions, taking as ground truth meta-analytic maps of language T-fMRI responses generated on the Neurosynth platform.

Results: Both fMRI methods localized major components of the language system (areas of Broca and Wernicke). Word-stem completion T-fMRI strongly activated Broca's area but also several task-general areas not specific to language. RS-fMRI provided a more specific representation of the language system.

Conclusion: 3DCNN was able to accurately localize the language network. Additionally, 3DCNN performance was remarkably tolerant of a limited quantity of RS-fMRI data.

Keywords: functional MRI, language, deep learning, resting state network, convolutional neural network

\section{INTRODUCTION}

In treating brain tumors, the neurosurgeon must balance the benefit of maximal tumor resection against the risk of a functional impairment consequent to more aggressive approaches. These two factors, maximal resection and functional preservation, are often cited in the surgical literature as predictors of long term survival (1-3). Thus, preoperative and intraoperative functional localization is critical to optimizing these often conflicting priorities. Functional MRI (fMRI) has been used as an adjunct measure for preoperative mapping of eloquent cortex and intraoperative navigation (4). The gold standard, however, for defining eloquent cortex is intraoperative mapping using electrical cortical stimulation mapping (5). Since electrocortical stimulation carries clinical risk (6), 
preoperative mapping to optimize the intraoperative surgical approach is an effective means of preserving function.

fMRI detects changes in the blood oxygen level dependent (BOLD) signal that reflect the neurovascular response to neural activity. In conventional fMRI, function is localized by presenting stimuli or imposing tasks (such as finger tapping or object naming) (7). More recently, resting state fMRI (RS-fMRI), i.e., fMRI obtained in the absence of stimuli or tasks, has been used to map the brain's functional organization (8). RSfMRI data is simpler to acquire and does not require patient cooperation (important in children and neurologically impaired patients). Thus, RS-fMRI has opened new opportunities for clinical applications, such as pre-surgical planning (9-11).

Multiple techniques have been used to map the representation of function using RS-fMRI data. These techniques include independent component analysis (12), seed-based correlation (13), and machine learning methods such as the multi-layer perceptron (MLP) (14). With typical fMRI acquisition times, these methods are signal to noise limited and have limited sensitivity and specificity. More recently, advances in deep convolutional neural networks $(\mathrm{CNN})$ have revolutionized image classification and segmentation problems $(15,16)$. It is thus logical to apply CNN methodology to the problem of mapping resting state networks.

In this study, we apply a CNN to RS-fMRI data for the localization of the language system in a cohort of patients with brain tumors and compare these results to the localization estimated in the same patients with task-based fMRI (TfMRI). We evaluated T-fMRI vs. RS-fMRI as methods for presurgical mapping of the representation of language. We used aggregated language T-fMRI data in the Neurosynth platform (www.neurosynth.org) (17) to define language localization ground truth. Our hypothesis is that the current study will provide preliminary data for the utility of $\mathrm{CNN}$ methods for the localization of the language system. We further wish to characterize differences in language localization between the CNN and T-fMRI methods.

\section{MATERIALS AND METHODS}

\section{Patients}

Patients were retrospectively recruited from the Neurosurgery brain tumor service at the Washington University School of Medicine in Saint Louis (WUSM), initially as part of an NIHfunded tumor data base grant (CONDR NIH 5R01NS066905). This patient cohort was used in a prior study targeting noninvasive localization of sensorimotor cortex (18). The following inclusion criteria were used: new diagnosis of primary brain tumor; age above 18 years; clinical need for an MRI scan including fMRI for presurgical planning as determined by the treating neurosurgeon. Additionally, we required that the patients have both a language task (word-stem completion) $\mathrm{T}$ fMRI and RS-fMRI. Exclusion criteria included: prior surgery for brain tumor, inability to have an MRI scan, or a patient referred from an outside institute with an MRI scan not performed at WUSM. Our cohort include $N=35$ patients (male/female 23/12) with a mean age of 44.8 years (23-71 years range). The mean preoperative enhancing tumor volume was $43.8 \mathrm{~mL}$ (range: $1.4-207 \mathrm{~mL}$ ); 28 patients had a left-hemisphere tumor; pathology was most often oligoastrocytoma (11 cases) and glioblastoma (10 cases). Handedness was recorded in 26 patients. To decrease any uncertainty in regard to laterality we also included the laterality index (LI) for all subjects based on (19). Since the 3 left handed patients had LI $>0$, and two of the three patients with LI $<0$ were right handed (the handedness on the third was not available) we opted to average language activation in all subjects as a single group. Patient demographics are summarized in Table 1. All aspects of the study were approved by the WUSM Institutional Review Board. Clinical data were acquired during preoperative evaluation and reviewed retrospectively.

\section{MRI Acquisition}

Patients were scanned with either a 3T Trio or Skyra scanner (Siemens, Erlangen, Germany) using a standard clinical presurgical tumor protocol. Anatomical imaging included T1-weighted (T1w) magnetization prepared rapid acquisition gradient echo (MP-RAGE), T2-weighted (T2w) fast spin echo, fluid-attenuated inversion recover (FLAIR), susceptibilityweighted imaging (SWI), and pre/post-contrast T1w fast spin echo in three projections. Additional sequences for presurgical mapping included Diffusion Tensor imaging (DTI) for track tracing, T-fMRI for motor and language localization, and RS-fMRI.

Both the task and resting state fMRI were acquired using echo planar imaging (EPI) (voxel size $3 \times 3 \times 3 \mathrm{~mm}$; TE $=27 \mathrm{~ms} ; \mathrm{TR}=2.2 \mathrm{~s}$; field of view $=256 \mathrm{~mm}$; flip angle $\left.=90^{\circ}\right)$. The language T-fMRI employed a block design in which patients covertly generated words in response to a visually presented first letter. Five task/rest blocks (10 frames each) were acquired over a total of 90-100 frames (3:40 min total per T-fMRI run). For most subjects, two language task sessions were acquired, and the run with the lowest root-meansquare head motion measure was used in the present analysis. RS-fMRI was acquired in two 160-frame runs (total of 320 frames $=11: 44 \mathrm{~min})$.

\section{Pre-processing}

The fMRI data were preprocessed using previously described techniques using locally written software (4dfp.readthedocs.io) (14). Preprocessing was identical for RS-fMRI and for T-fMRI and included compensation for slice dependent time shifts, elimination of systemic odd-even slice intensity differences due to interleaved acquisition, and rigid body correction for head movement within and across runs. Atlas transformation was achieved by composition of affine transforms connecting the fMRI volumes with the T2-weighted and MPRAGE structural images, resulting in a volumetric time series in ( $3 \mathrm{~mm}$ cubic) atlas space. Additional preprocessing included: spatial smoothing (6 mm full width half maximum Gaussian blur in each direction), voxelwise removal of linear trends over each run, and temporal low pass filtering retaining frequencies $<0.1 \mathrm{~Hz}$. Spurious variance was reduced by regression of nuisance waveforms derived from head motion correction and extraction of the 
TABLE 1 | Patient clinical and demographic data.

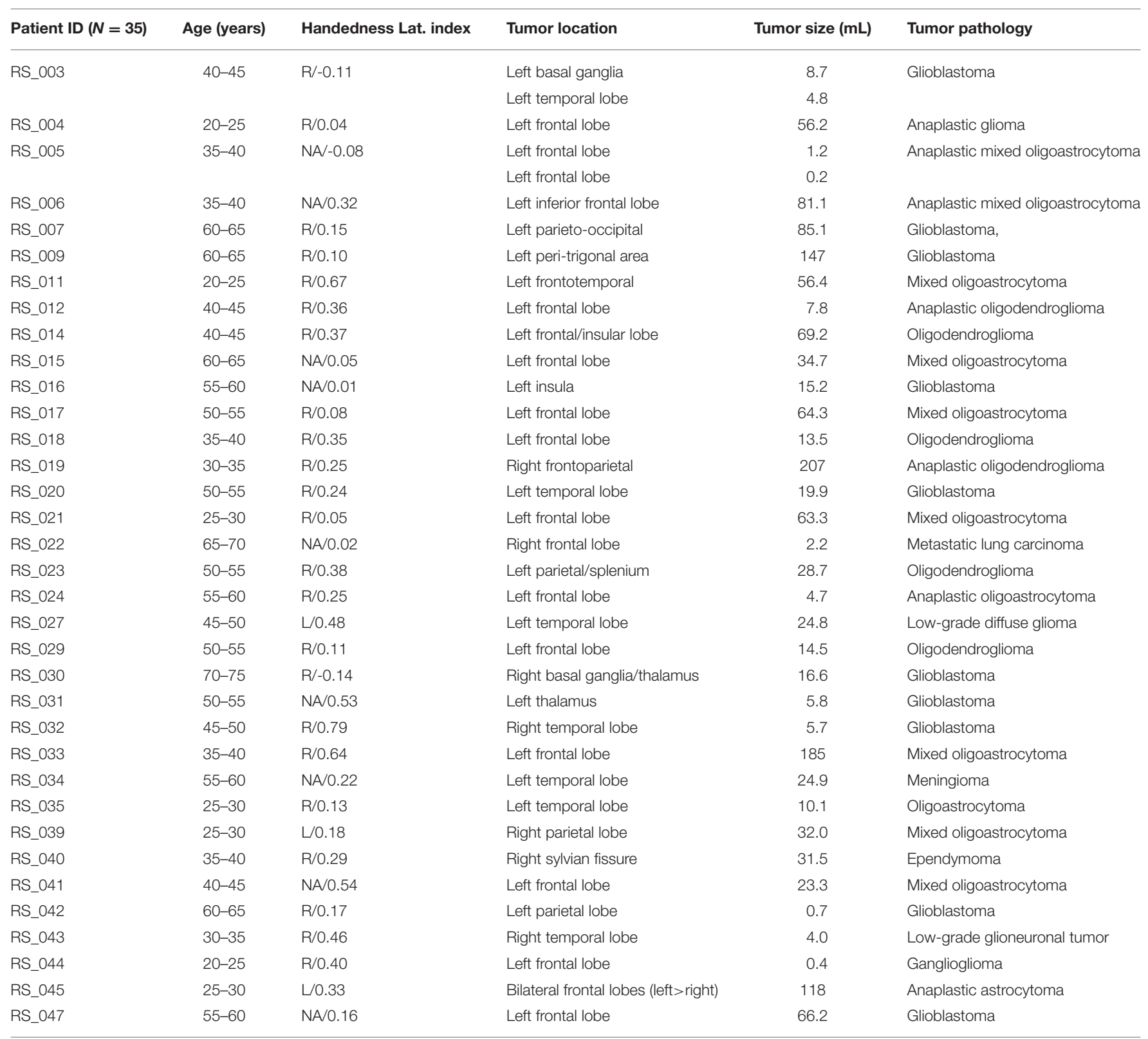

Clinical data for 35 patients with brain tumors (age $44.8 \pm 14.0$ years; 12 female).

time series from regions of white matter and CSF. The whole brain ("global") signal was included as a nuisance regressor (20). Frame censoring was performed to minimize the impact of head motion on the correlation results (21). Thus, frames (volumes) in which the root mean square (evaluated over the whole brain) change in voxel intensity relative to the previous frame exceeded $0.5 \%$ (relative to the whole brain mean) were excluded from the functional connectivity computations (22). All fMRI data acquired in each patient were pooled during preprocessing. Thus, the T-fMRI and RS-fMRI data were mutually co-registered in each patient. Additionally, all T-fMRI and RS-fMRI data in all patients were resampled in a standard atlas space. No attempt was made to correct for the mass effect of tumors. To match acquisition durations of RS-fMRI and T-fMRI (11:44 vs. 3:40 min), we selected 100 contiguous frames from pre-processed RS-fMRI data for comparisons with T-fMRI. Additionally, the full quantity of RS-fMRI data was compared to T-fMRI. T-fMRI responses were evaluated using standard general linear model methods using in house software. Activation maps were generated from the T-fMRI as described in Corbetta et al. (23), smoothed with a $10 \mathrm{~mm}$ Gaussian filter, and masked to exclude extracranial voxels. Neither response clustering nor thresholding was done. 


\section{Deep Learning-Convolutional Neural Networks Processing \\ Training}

Normal human resting state fMRI data $(N=2,795)$ were obtained from the Brain Genomics Superstruct Project (GSP) (Harvard University) (24) and ongoing studies at Washington University in St. Louis including the Alzheimer's Disease Research Center (ADRC) (25), the Dominantly Inherited Alzheimer's Network (DIAN) (26), and studies by the Division of Infectious Diseases HIV Program (HIV) (27) (Table 2). Statistical analysis of network FC (evaluated within and across the default mode network, the dorsal attention network, vision network, and deep gray structures) between the different data sets revealed no significant group effect attributable to study. Each subject had $\sim 14 \mathrm{~min}$ of resting state fMRI data (TR $=3,000 \mathrm{~ms}, 3 \mathrm{~mm}$ cubic) which was processed using standard methods developed at WUSM (14). Resting state networks (RSNs) were identified using a set of 169 region of interests (ROI) divided into 11 RSNs (14). Multiple $(n=268,000)$ example sets were generated from the data and then divided into a training $(N=18,7600)$ and validation $(N=80,400)$ sets. A 3D convolutional neural network (3DCNN) with a densely connected architecture (15) was trained to classify brain regions as belonging to a priori assigned RSNs. The 3DCNN consisted of 49 layers and 3 dense blocks that performed 3 and 5 cubic convolutions. Batch normalization was used within the network to prevent overfitting and improve performance, and average pooling was used for dimensionality reduction. Training was terminated if the accuracy did not improve after 3 validations. The 3DCNN was implemented in Matlab R2019b (www.mathworks.com).

\section{Testing}

For each of 35 tumor patients the T-fMRI results were compared to the $3 \mathrm{DCNN}$ results obtained with matched data samples, i.e., 100 contiguous frames of RS-fMRI. Additionally, the 3DCNN analysis was run using all available data (320 RS-fMRI frames per subject). 3DCNN maps representing the probability of language

TABLE 2 | Studies used to obtain normal training data.

\begin{tabular}{|c|c|c|c|c|}
\hline & GSP & ADRC & DIAN & HIV \\
\hline N & 1,137 & 1,289 & 336 & 775 \\
\hline Age (std) & $21.4(2.4)$ & $68.1(7.9)$ & $40.9(10.9)$ & $44.3(16.3)$ \\
\hline Scanner & Trio & $\begin{array}{c}\text { Trio/ } \\
\text { Biograph }\end{array}$ & $\begin{array}{l}\text { Trio/ } \\
\text { Verio }\end{array}$ & $\begin{array}{l}\text { Trio/ } \\
\text { Prisma }\end{array}$ \\
\hline $\begin{array}{l}\text { Voxel Size in cubic } \\
\mathrm{mm}\end{array}$ & 3.0 & 4.0 & 3.3 & 4.0 \\
\hline Flip angle in degrees & 85 & 90 & 80 & 90 \\
\hline $\begin{array}{l}\text { Repetition time (TR) } \\
\text { in ms }\end{array}$ & 3,000 & 2,200 & 3,000 & 2,200 \\
\hline $\begin{array}{l}\text { Total number of } \mathrm{fMRI} \\
\text { frames }\end{array}$ & 248 & 328 & 140 & 328 \\
\hline
\end{tabular}

Refer to text for acronyms and citations. representation were smoothed with stride- 1 mode filtering and length-3 box filtering.

\section{A Priori Defined Language Regions of Interest (ROI)}

Language representation in the brain resides primarily on two areas of the left cerebral cortex: Broca's area, located in inferior frontal cortex (roughly, Brodmann areas 44 and 45) and frontal operculum (28), is required for fluid performance of phonemic or semantic tasks (29). Wernicke's area extends over portions of temporal and parietal cortex and is essential for understanding written or spoken language (30). The Broca-Wernicke model embodies core expressive and receptive language functions but omits auxiliary functions such as reading (31-33).

We defined the ground truth for language representation using T-fMRI responses aggregated by Neurosynth (17). This representation was confined to the left hemisphere to simplify comparison between the Neurosynth regions and those derived in our patients. To define T-fMRI-based language ROIs, we queried Neurosynth using "language comprehension" as a search term, which identified 107 studies (as of November, 2018) contributing coordinates in Talairach atlas space, each coordinate associated with a Z-score corresponding to the null hypothesis of equally likely activation anywhere in the brain. The returned association map (threshold at $\mathrm{Z}>3.7$ by Neurosynth) was passed through smoothing and clustering operations (see below), ultimately yielding Broca- and Wernicke-like ROIs in volumetric atlas space (Figure 1A).

The following steps were taken to obtain Broca- and Wernicke-like ROIs starting with a "language comprehension" map (units $=Z$-score) generated by Neurosynth in 2 cubic $\mathrm{mm}$ MNI152 atlas space:

1. Gaussian smooth using a kernel of $1 \mathrm{~mm}$ full width at half maximum (FWHM) in each cardinal direction.

2. Transform Z-scores $>0$ to probability maps using the hyperbolic tangent and threshold at Z-score $>3.7$.

3. Retain two largest clusters to generate initial estimates of Broca and Wernicke regions in the left hemisphere.

4. Gaussian smooth using a $3 \mathrm{~mm}$ FWHM isotropic kernel in each cardinal direction.

5. Resolve overlapping clusters into two disjoint ROIs by assigning multiply labeled voxels to the ROI with the nearest center of mass.

\section{Image Computation and Visualization Software}

fMRI preprocessing, denoising, and computation of RS-fMRI and T-fMRI responses were done using the 4dfp software library (https://readthedocs.org/projects/4dfp/). MATLAB R2019b was used for statistical computations and visualization. Connectome Workbench, version 1.2.3 (www.humanconnectome.org), was used to map volumetric data onto the PALS-B12 midthickness surfaces and rendered on the corresponding inflated surface (34). 


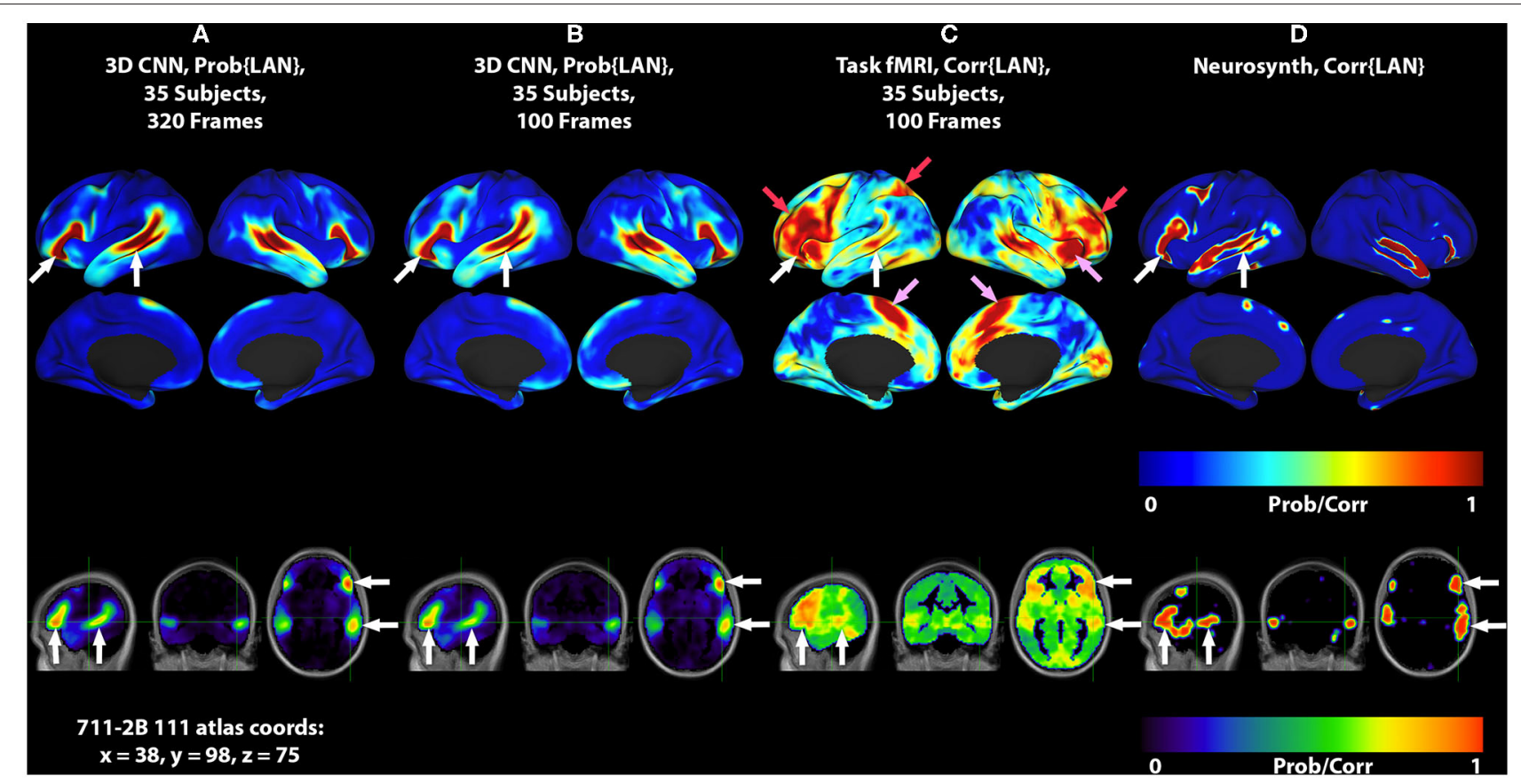

FIGURE 1 | 3DCNN maps and T-fMRI responses focusing on language localization. All RS-fMRI and T-fMRI results are averages over 35 patients. Surface plots show probabilities thresholded at $p>0.02$. Top row shows lateral surface plots; middle row shows medial surfaces; bottom row shows sagittal, coronal, and axial views at coordinate $\mathrm{x}=38, \mathrm{y}=98, \mathrm{z}=75$ on the $711-2 \mathrm{~B}$ atlas using radiologic conventions (left body on right image). Columns show: (A) 3DCNN language (LAN) map computed using 320 frames per patient (all available RS-fMRI data). (B) 3DCNN LAN map computed with only 100 frames per patient. (C) Word stem completion T-fMRI responses. (D) Neurosynth map derived with the search term, "language comprehension." White arrows indicate the areas of Broca and Wernicke. Pink arrows indicate task responses in the right anterior insula and dorsal anterior cingulate cortex (core task-control regions). Red arrows indicate task responses in antero-lateral prefrontal cortex and superior parietal lobule (dorsal attention and fronto-pariental control networks).

\section{Statistical Analysis}

RS-fMRI and T-fMRI produce native measurements with distinct statistical properties. Acceptable conventions for image processing, denoising, and significance testing have evolved distinctly for these functional imaging methods. Additionally, highly non-linear deep learning architectures such as 3DCNNs have unknown statistical properties when applied to functional imaging data. To enable meaningful statistical comparisons in our data, we used probabilistic strategies to enhance the detection of the population-invariant language network and reduce the influence of experimental conventions, differential preprocessing and the biological variability arising from the use of normal training data but pathophysiologic testing data. Specifically, we reused common image processing pipelines wherever possible in our analyses. We reduced all methodspecific metrics to normalized probability maps. For purposes of group-level inferences, for each patient, all temporal imaging information was contracted into T-fMRI activations or RSfMRI membership in RSNs. As described in the preprocessing methods, all T-fMRI and RS-fMRI data were co-registered to a standardized atlas. Consequently, spatially distributed measures of task activation or resting state network membership retained co-registration in atlas space. We used arithmetic averages of patient data prior to computing comparative analyses at the group-level. Finally, we used method-dependent thresholds for detection of language networks by analysis of receiver operating characteristics (ROC). ROC computations made exclusive use of the perfcurve method from Matlab. Significance testing at alpha $=0.05$ included computation of point-wise confidence intervals on true positive rates by vertical averaging over 101 false positive rate intervals and resampling with 10,000 bootstrap iterations. All other parameterizations of perfcurve were default values.

\section{RESULTS}

\section{Mean Language Maps}

Figure 1 shows group level localizations of the language network. Figures 1A,B are computed from 3DCNN analysis of RS-fMRI, using the full amount of available resting state data $(1 \mathrm{~A})$, and one third of the available data (1B), comparable to the amount of data available in the T-fMRI. Figure 1C is a group level language map computed from the T-fMRI response to wordstem completion. Figure 1D is derived from the Neurosynth platform (17) using the search term "language comprehension." Both T-fMRI and RS-fMRI clearly identify Broca and Wernicke regions. The 3DCNN method provides highly specific maps with large probability gradients at the margin of the language regions, as would be expected of a method trained on thousands of exemplars including millions of internal parameters. The TfMRI experiment focused on expressive language and therefore 
emphasizes Broca's region. The $3 \mathrm{DCNN}$ map reflects the properties of spontaneous activity which characteristically is more symmetric than task responses. Robust delineation of both Broca's and Wernicke's area is not surprising as the 3DCNN was trained to recover the topography of T-fMRI responses in RS-fMRI data (14).

The crucial difference between the two methods is that the word stem completion task activates areas not specifically associated with language (pink and red arrows in Figures 1, 2) in addition to areas that are specifically associated with language (pink arrows in Figures 1, 2). Task-general responses occur in the cingulo-opercular network (35), the dorsal attention system (23), and fronto-parietal control network (36). Additional discussion of these task-general systems is given below.

\section{Correspondence of Identified Language Maps With Neurosynth Reference}

We assessed the topography of T-fMRI and RS-fMRI maps in relation to language ROIs defined on the basis of aggregated fMRI responses to language tasks (Figure 1D). ROC curves for Broca's area are presented in Figure 3A, and for Wernicke's area in Figure 3B.

In Broca's area, the 3DCNN AUC exceeded that for T-fMRI for both lengths of data, full length AUC $=0.9516$ [0.9469, 0.9556] vs. 0.9100 [0.8992 0.9196] and for 100 frame data $\mathrm{AUC}=0.9545$ [0.9502, 0.9587] vs. 0.9100 [0.8992, 0.9196]. Notably, the AUC between the 3DCNN full length data and that for the shortened 100 frame data had overlapping 95\% confidence intervals.

In Wernicke's area, the differences between the 3DCNN AUC and that of the T-fMRI was much larger, full length AUC $=0.9490$ $[0.9450,0.9527]$ vs. $0.6679[0.6549,0.6811]$ and for 100 frame data $\mathrm{AUC}=0.9495[0.9449,0.9537]$ vs. $0.6679[0.6549,0.6811]$. As in the Broca's case, the AUC between the 3DCNN full length data and that for the shortened 100 frame data had overlapping $95 \%$ confidence intervals.

\section{Case Examples}

This section demonstrates two case examples of the ability of the 3DCNN method to provide data acquired in individual patients and a comparison between the 3DCNN method and the T-fMRI at the individual level. Individual cases took approximately $4 \mathrm{~h}$ of computation time on a Dell (Austin, Texas) Power Edge 18 core with Nvidia (Santa Clara, California) v100 GPU.

\section{Case 1}

Images from a 44 year old right handed male (RS003) with glioblastoma multiform in the left basal ganglia region are presented in Figure 4. The top two rows display the anatomy with a post contrast T1-weighted and FLAIR images. The bottom two rows display the language localization information from the 3DCNN and T-fMRI overlying the anatomical images. We provide the T-fMRI at several thresholds in accordance with clinical practice. Although the T-fMRI appears noisier (bottom row) than the 3DCNN (third row), the information provided by both methods is similar with significant overlap of the localized language area with the tumor location.

\section{Case 2}

Images from a 24 years old right handed male (RS004) with anaplastic glioma in the subcortical left frontal lobe are presented in Figure 5. As in Case 1, The top two rows display the anatomy with a post contrast T1-weighted and FLAIR images. The bottom two rows display the language localization information from the $3 \mathrm{DCNN}$ and T-fMRI overlying the anatomical images. In this case, the sagittal views of the two methods are very similar, although the $3 \mathrm{DCNN}$ (third row) demonstrates more overlap of the language localization with the tumor as compared to the T-fMRI (bottom row).

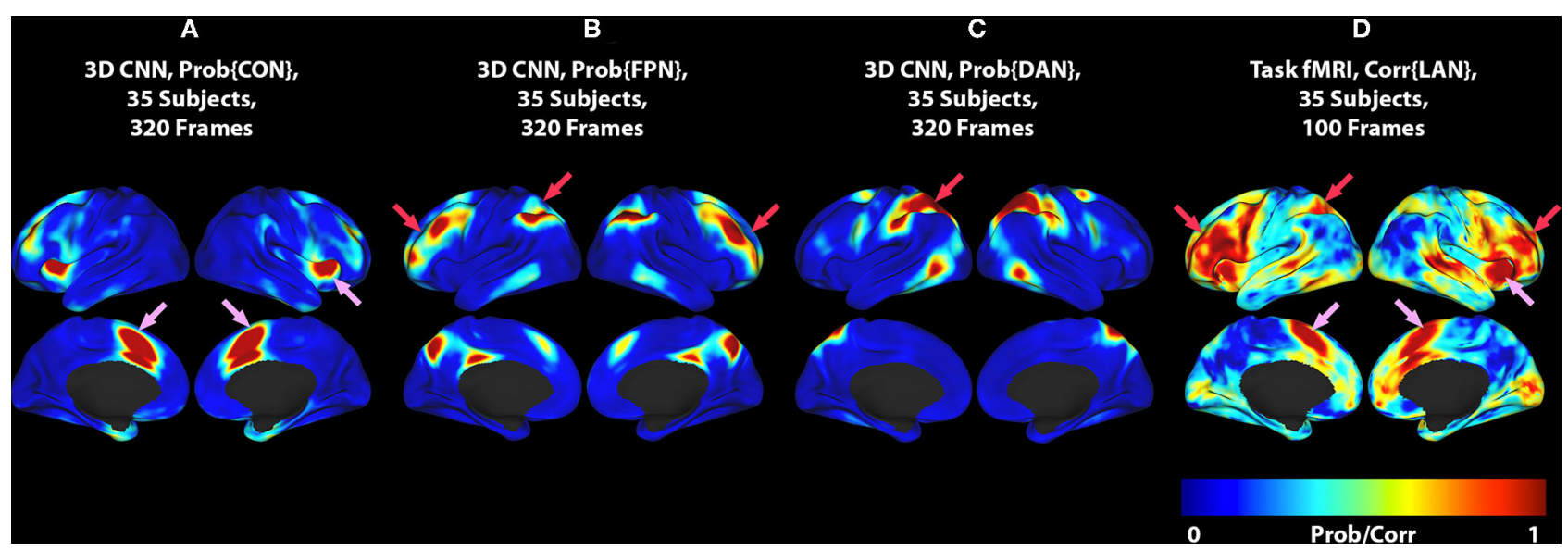

FIGURE 2 | (A) 3DCNN map of the CON. (B) 3DCNN map of the FPN. (C) 3DCNN map of the DAN. (D) T-fMRI responses (reproduced from Figure 1C). Pink arrows point to components of the cingulo-opercular network (CON). Red arrows point to components of the dorsal attention network (DAN) and fronto-parietal control network (FPC). See text for discussion of these task-general functional systems. 


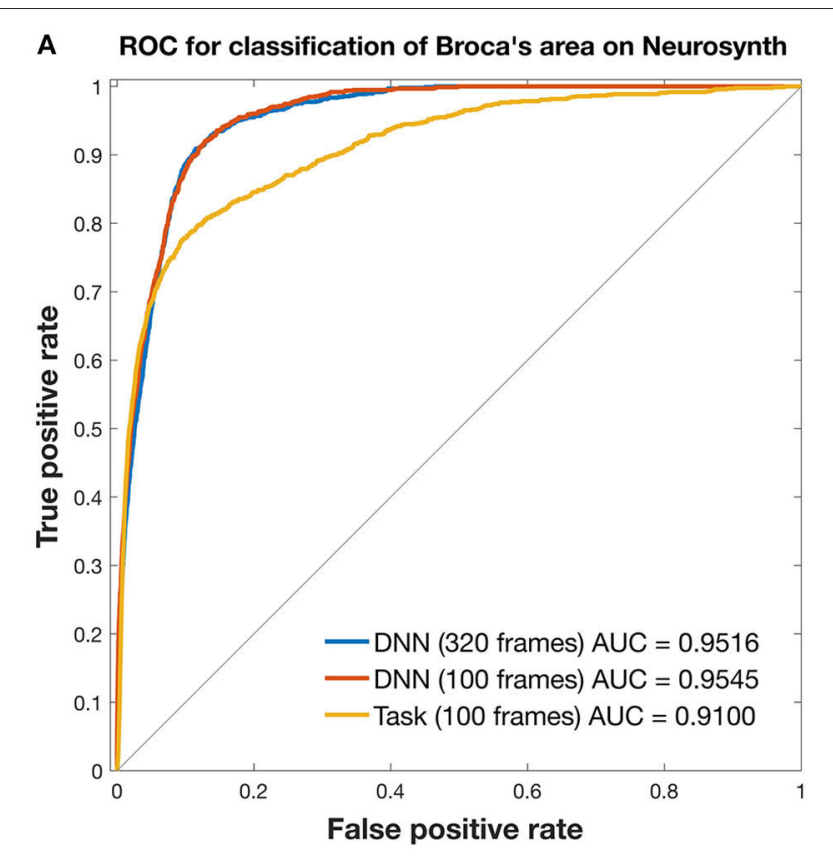

B ROC for classification of Wernicke's area on Neurosynth

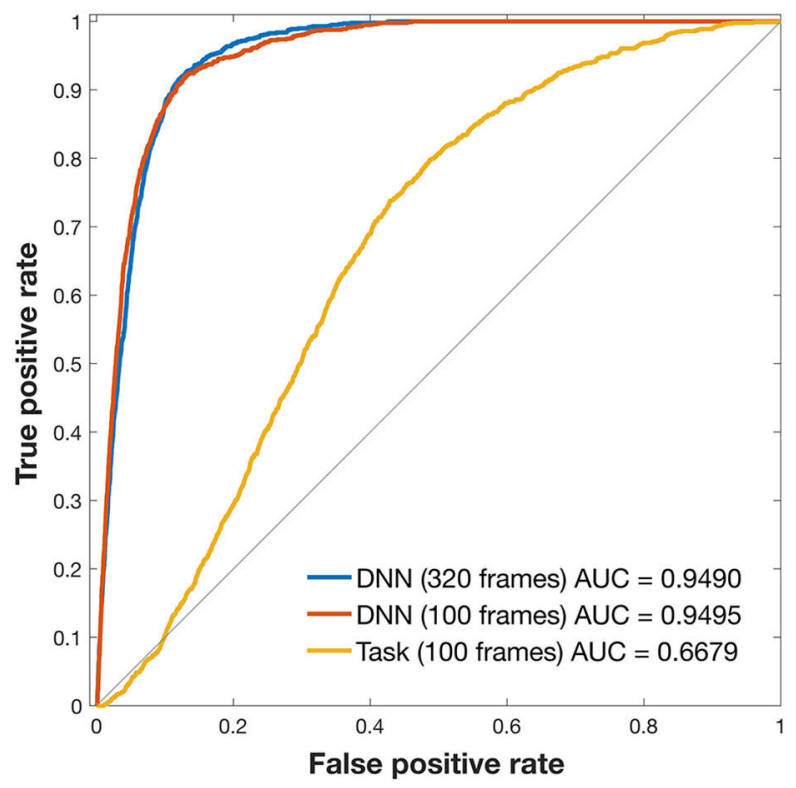

FIGURE 3 | ROC curves for mapping the language network. Curves are from 3DCNN using 320 (blue) or 100 (red) resting-state fMRI frames. Additional curves are from 100 (yellow) T-fMRI frames. Ground truth labels are binary classes derived from thresholded Neurosynth data. All curves were constructed after averaging over 35 brain tumor patients. (A) Broca's area and (B) Wernicke's area are assessed separately. See text for details.

\section{DISCUSSION}

The current work demonstrates that the representation of language in the brain can be identified using 3DCNN analysis of RS-fMRI data (Figure 1, white arrows). It should be noted that the $3 \mathrm{DCNN}$ was trained to identify language-associated parts of the brain using T-fMRI acquired at Washington University School of Medicine [same training set used by (14)]. The Neurosynth-derived map was obtained from an independent meta-analysis of 107 reported neuroimaging studies. Nevertheless, the 3DCNN and Neurosynth maps are strikingly similar.

The differences between T-fMRI and 3DCNN maps are instructive. The word-stem completion task activated the dorsal anterior cingulate (dACC) (a.k.a the rostral cingulate zone) as well as the right anterior insula (Figures 1, 2, pink arrows). These regions are components of the salience network (37), also known as core task-control regions $(35,38)$. The core task control system is recruited by a wide variety of goal-directed behaviors (38-40). Functions attributed to the dACC include task control $(41,42)$, error monitoring (43), and conflict detection (44). Additional T-fMRI responses not specific to the LAN occurred in the left superior parietal lobule and the left middle frontal gyrus. These regions are components of the dorsal attention network (DAN) and the fronto-parietal control network (FPC) (Figures 1, 2, red arrows). The DAN responds to any task requiring directed spatial attention (23, 45-47). The FPC supports goal-directed analysis of environmental stimuli (48-50). These functional systems are recruited by the word stem completion task as it requires directing attention to and analyzing stimuli presented on an electronic display.

The present results raise the possibility of distinguishing between parts of the brain that are language specific (28) vs. task-general (38-40). This distinction may be of value in selected neurosurgical cases. Although the DAN and dACC are not conventionally classified as "eloquent" (51), injury to these areas can lead to attentional deficits (52) and to loss of motivated behaviors (53), respectively.

An additional important observation evident in Figure $\mathbf{1}$ is that the localization of the language network using the 3DCNN appears remarkably tolerant to limited quantities of RS-fRMI data (Figures 1A,B). This characteristic could lead to reduced RS-fMRI acquisition times.

Figure 3 compares T-fMRI vs. 3DCNN as regards localization of Broca and Wernicke areas as defined a priori, according to a large collection of T-fMRI studies aggregated by Neurosynth (17). According to the AUC measure, 3DCNN had a small but significant advantage over T-fMRI in localizing Broca's area (Figure 3A). The difference was much larger in Wernicke's area (Figure 3B). This result is understandable as the word stem completion task is an expressive language task that preferentially activates Broca's area. Performing several different T-fMRI studies better characterizes the language system (54-56). Figure 3 also demonstrates 3DCNN tolerance to a limited quantity of data: there is no significant difference in AUC corresponding to $100 \mathrm{vs.}$ 320 frames (red and blue curves).

The case examples demonstrate 3DCNN functional mapping in individual patients with brain tumors (Figures 4, 5). The 


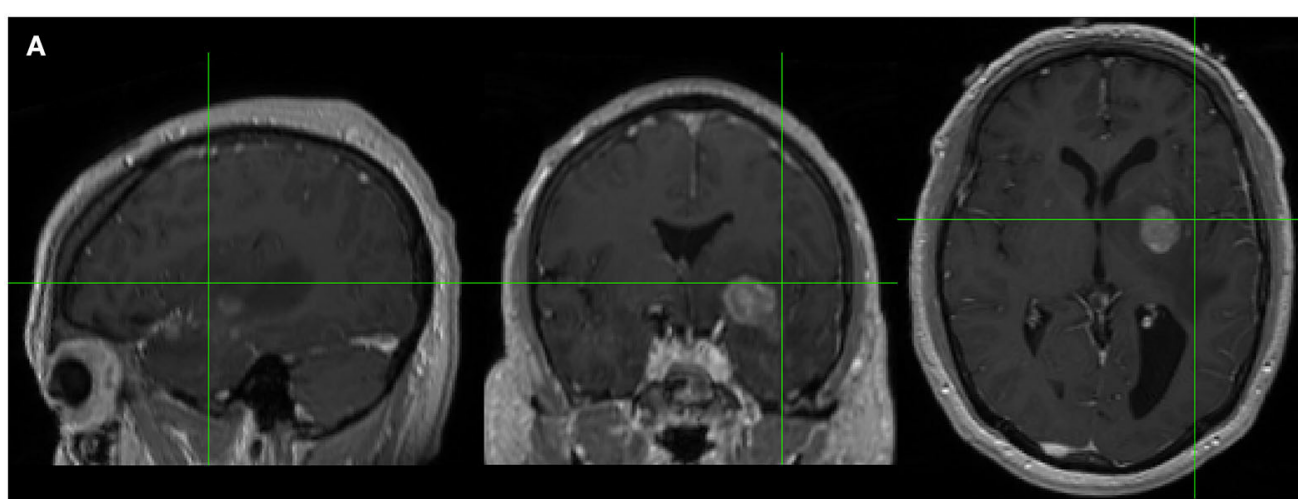

B

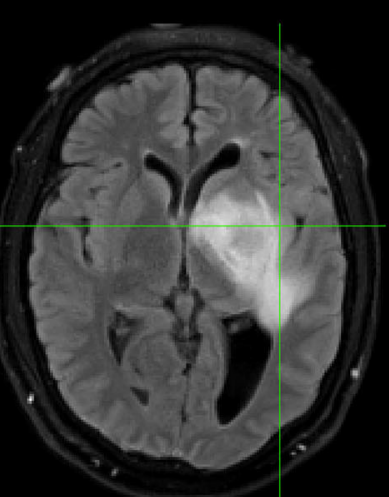

C
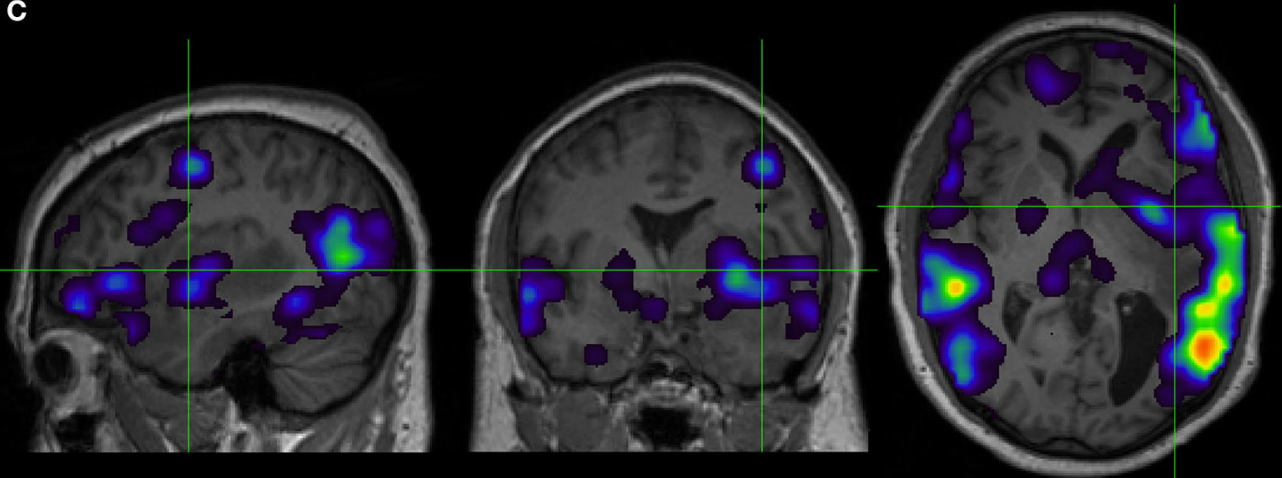

D

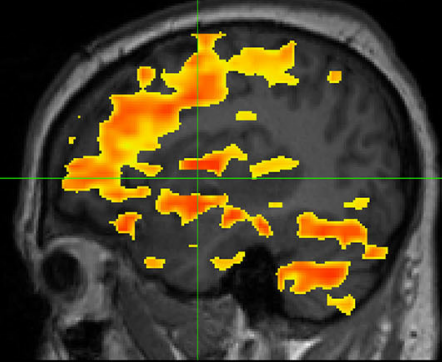

threshold $=0.7$

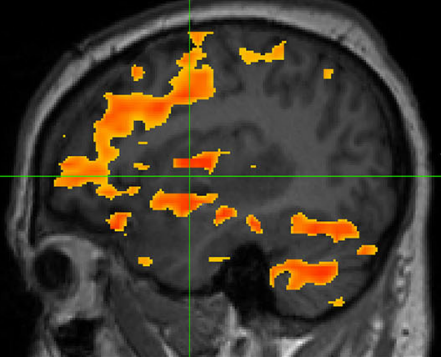

threshold $=0.8$

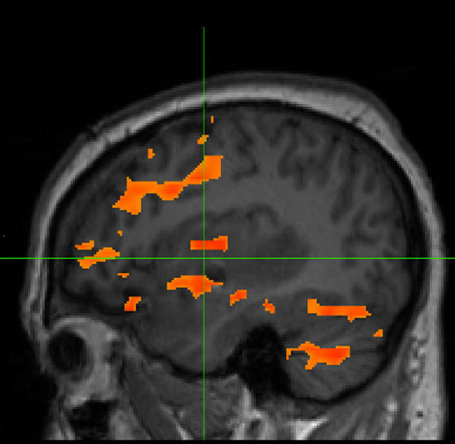

threshold $=0.9$

FIGURE 4 | Exemplar patient RS003 with glioblastoma multiforme in the left basal ganglia region. (A) Contrast-enhanced T1 and (B) fluid-attenuated inversion recovery demonstrating the tumor and surrounding edema. (C) Probability map of language network from 3DCNN with significant overlap of the tumor and (D) probability map of language from the T-fMRI also showing overlap over the tumor. Deep learning results show probabilities $>0.02$. Task fMRI thresholds are varied in accordance with clinical practice. 


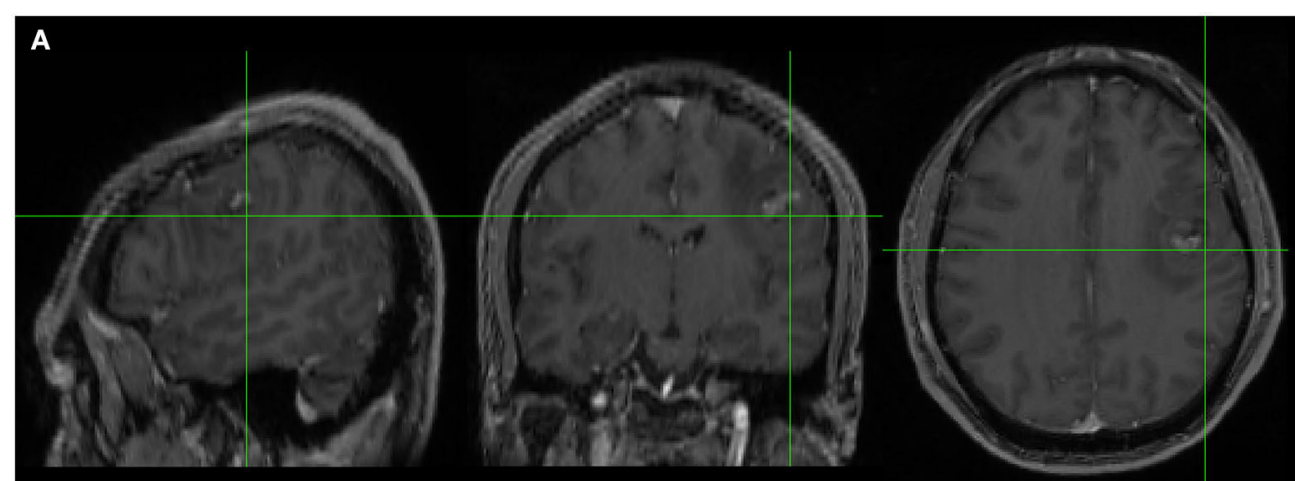

B

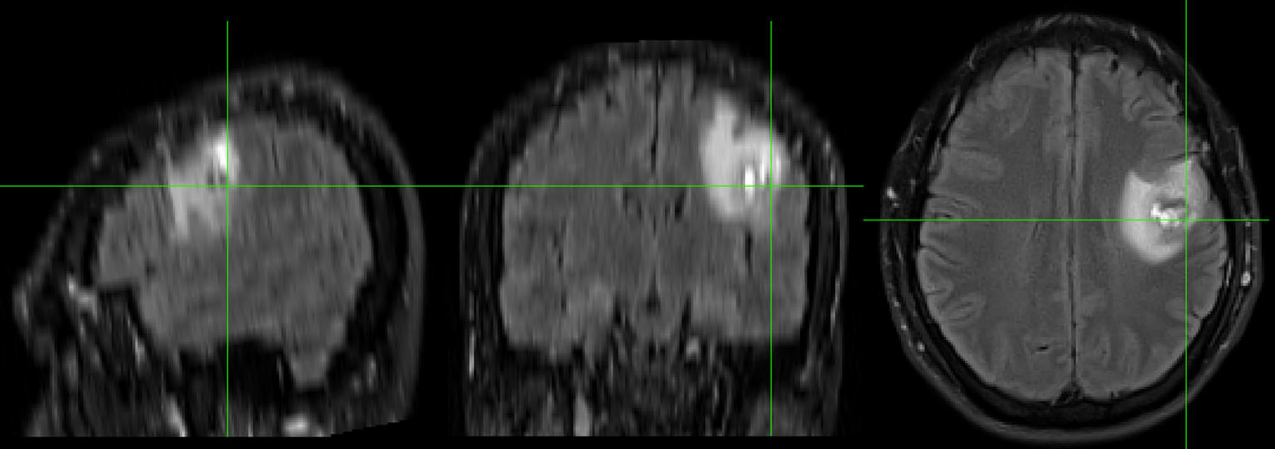

C

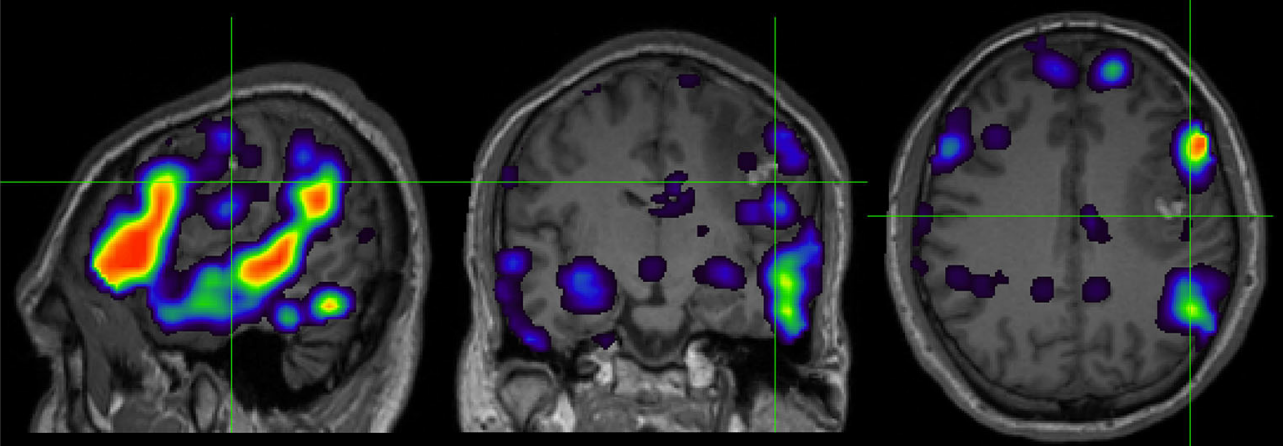

D

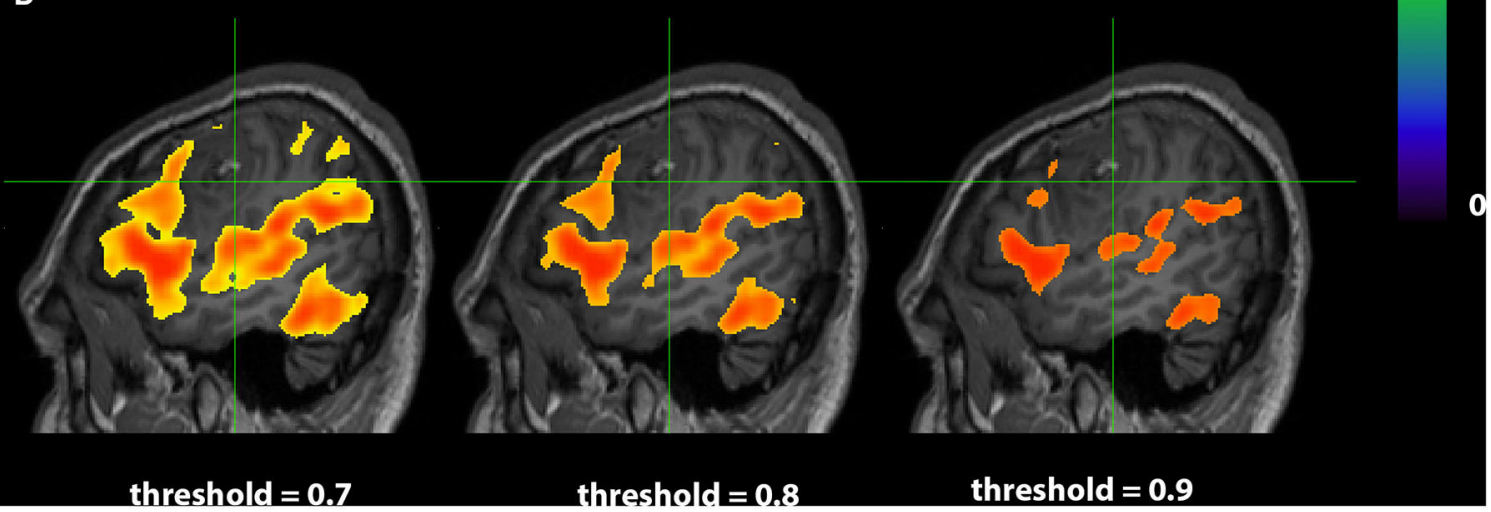

FIGURE 5 | Exemplar patient RS004 with high grade glioma with anaplastic features in the subcortical left frontal lobe. (A) Contrast-enhanced T1 and (B) fluid-attenuated inversion recovery demonstrating the tumor and surrounding edema. (C) Probability map of language network from 3DCNN with mild overlap of the tumor and (D) probability map of language from the T-fMRI also showing mild overlap over the tumor. Deep learning results show probabilities $>0.02$. Task fMRI thresholds are varied in accordance with clinical practice. 
higher specificity and sharper margins of the 3DCNN method in comparison to T-fRMI is promising. A prospective comparison of the 3DCNN RS-fRMI method vs. T-fMRI remains to be done.

Limitations of our study include first, that it is formulated in terms of group-level analyses; hence, our results do not directly speak to the question of which technique provides the best functional localization of language in individuals. Our focus has been on regions instantiating expressive and receptive language functions (Broca and Wernicke). Further study will be needed to evaluate the utility of classifier-based analysis or RS-fMRI for localizing functions such as reading, articulation, and prosody. Additionally, we make no claims for the $3 \mathrm{DCNN}$ in determining language lateralization. As our data are retrospective, we have no means of assessing the potential effects of neuro-vascular uncoupling (57). Similarly, we have not considered how anatomical distortion owing to tumor mass may have affected our results, although the average tumor size was small ( $\sim 44 \mathrm{~mL})$. This work omits comparison with intraoperative brain mapping; that comparison is reported in prior related work (58). The relatively poor performance of T-fMRI in localizing Wernicke's area is a limitation of the word stem completion task. This limitation could be overcome by use of a wider range of language tasks $(33,56)$. Distorted brain anatomy in tumor patients compromises affine atlas registration; however, this issue will have affected T-fMRI and 3DCNN equally. Finally, a definitive comparison of RS-fMRI vs. 3DCNN in terms of patient outcomes would require a prospective, multi-center, clinical trial (59).

\section{CONCLUSION}

This study demonstrates that 3DCNN analysis of RS-fMRI data is able to accurately and specifically localize the language network in patients with brain tumors. In addition to the inherent advantages of RS-fMRI, specifically, limited requirement for patient cooperation, the $3 \mathrm{DCNN}$ method provides robust results with limited quantities of data, which is an advantage in the clinical setting. We anticipate that this method will lead to improved pre-surgical localization in future applications.

\section{REFERENCES}

1. Lacroix M, Abi-Said D, Fourney DR, Gokaslan ZL, Shi W, DeMonte F, et al. A multivariate analysis of 416 patients with glioblastoma multiforme: prognosis, extent of resection, and survival. J Neurosurg. (2001) 95:190-8. doi: 10.3171/jns.2001.95.2.0190

2. McGirt MJ, Chaichana KL, Gathinji M, Attenello FJ, Than K, Olivi A, et al. Independent association of extent of resection with survival in patients with malignant brain astrocytoma. J Neurosurg. (2009) 110:156-62. doi: 10.3171/2008.4.17536

3. Gulati S, Jakola AS, Nerland US, Weber C, Solheim O. The risk of getting worse: surgically acquired deficits, perioperative complications, and functional outcomes after primary resection of glioblastoma. World Neurosurg. (2011) 76:572-9. doi: 10.1016/j.wneu.2011.06.014

4. Petrella JR, Shah LM, Harris KM, Friedman AH, George TM, Sampson $\mathrm{JH}$, et al. Preoperative functional MR imaging localization of language

\section{DATA AVAILABILITY STATEMENT}

The datasets generated for this study are available on request to the corresponding author.

\section{ETHICS STATEMENT}

The studies involving human participants were reviewed and approved by Washington University Human Research Protection Office. The patients/participants provided their written informed consent to participate in this study.

\section{AUTHOR CONTRIBUTIONS}

JS, PL, JL, KP, and CH: conceptualization and methodology. $\mathrm{DD}, \mathrm{KP}, \mathrm{JL}, \mathrm{PL}$, and $\mathrm{AD}$ : data curation. JL, PL, KP, CH, and BS: formal analysis. EL and JS: funding acquisition. EL, JS, JL, and PL: investigation. EL, JS, and BA: project administration, supervision, and resources. PL, JL, KP, CH, and AS: software. PL and JL: validation. DD, JL, and BS: visualization. JS, AS, and KP: writing original draft. AS, JS, BS, JL, PL, EL, BA, DD, AD, and $\mathrm{CH}$ : writing review/editing. All authors contributed to the article and approved the submitted version.

\section{FUNDING}

Funding was provided by the National Institute of Health via NIH R01 CA203861. JS was additionally supported by the Eunice Kennedy Shriver National Institute of Child Health \& Human Development of the National Institutes of Health under Award Number U54 HD087011 to the Intellectual and Developmental Disabilities Research Center at Washington University. AS was supported by P30 NS098577-01. EL was additionally supported by the Christopher Davidson Foundation. AD was supported by the Chancellor's Graduate Fellowship. Computations were performed using the facilities of the Washington University Center for High Performance Computing, which were partially provided through NIH grant S10 OD018091. and motor areas: effect on therapeutic decision making in patients with potentially resectable brain tumors. Radiology. (2006) 240:793-802. doi: 10.1148/radiol.2403051153

5. Ojemann GA. Functional mapping of cortical language areas in adults. Intraoperative approaches Adv Neurol. (1993) 63:155-63.

6. Szelenyi A, Bello L, Duffau H, Fava E, Feigl GC, Galanda M, et al. Intraoperative electrical stimulation in awake craniotomy: methodological aspects of current practice. Neurosurg Focus. (2010) 28:E7. doi: 10.3171/2009.12.FOCUS09237

7. Spitzer M, Kwong KK, Kennedy W, Rosen BR, Belliveau JW. Categoryspecific brain activation in fMRI during picture naming. Neuroreport. (1995) 6:2109-12. doi: 10.1097/00001756-199511000-00003

8. Biswal B, Yetkin FZ, Haughton VM, Hyde JS. Functional connectivity in the motor cortex of resting human brain using echo-planar MRI. Magn Reson Med. (1995) 34:537-41. doi: 10.1002/mrm.19103 40409 
9. Kokkonen SM, Nikkinen J, Remes J, Kantola J, Starck T, Haapea M, et al. Preoperative localization of the sensorimotor area using independent component analysis of resting-state fMRI. Magn Reson Imaging. (2009) 27:733-40. doi: 10.1016/j.mri.2008.11.002

10. Liu H, Buckner RL, Talukdar T, Tanaka N, Madsen JR, Stufflebeam SM. Task-free presurgical mapping using functional magnetic resonance imaging intrinsic activity. J Neurosurg. (2009) 111:746-54. doi: $10.3171 / 2008.10$.JNS08846

11. Shimony JS, Zhang D, Johnston JM, Fox MD, Roy A, Leuthardt EC. Resting-state spontaneous fluctuations in brain activity: a new paradigm for presurgical planning using fMRI. Acad Radiol. (2009) 16:578-83. doi: 10.1016/j.acra.2009.02.001

12. Smith SM, Fox PT, Miller KL, Glahn DC, Fox PM, Mackay CE, et al. Correspondence of the brain's functional architecture during activation and rest. Proc Natl Acad Sci USA. (2009) 106:13040-5. doi: 10.1073/pnas.0905267106

13. Zhang D, Johnston JM, Fox MD, Leuthardt EC, Grubb RL, Chicoine $\mathrm{MR}$, et al. Preoperative sensorimotor mapping in brain tumor patients using spontaneous fluctuations in neuronal activity imaged with functional magnetic resonance imaging: initial experience. Neurosurgery. (2009) 65:22636. doi: 10.1227/01.NEU.0000350868.95634.CA

14. Hacker CD, Laumann TO, Szrama NP, Baldassarre A, Snyder AZ, Leuthardt EC, et al. Resting state network estimation in individual subjects. Neuroimage. (2013) 82:616-33. doi: 10.1016/j.neuroimage.2013.05.108

15. Huang G, Liu Z, Van Der Maaten L, Weinberger K. Densly connected convolutional networks. In: The IEEE Conference on Computer Vision and Pattern Recognition (CVPR). Honolulu, HI (2017). doi: 10.1109/CVPR.2017.243

16. Chen L, Zhu Y, Papandreou G, Schroff F, Adam H. Encoderdecoder with atrous separable convolution for semantic image segmentation. In: V. Ferrari, editor. ECCV 2018. Springer Nature (2018). doi: 10.1007/978-3-030-01234-2_49

17. Yarkoni T, Poldrack RA, Nichols TE, Van Essen DC, Wager TD. Large-scale automated synthesis of human functional neuroimaging data. Nat Methods. (2011) 8:665-70. doi: 10.1038/nmeth.1635

18. Dierker D, Roland JL, Kamran M, Rutlin J, Hacker CD, Marcus DS, et al. Resting-state functional magnetic resonance imaging in presurgical functional mapping: sensorimotor localization. Neuroimaging Clin N Am. (2017) 27:62133. doi: 10.1016/j.nic.2017.06.011

19. Wilke M, Lidzba K. LI-tool: a new toolbox to assess lateralization in functional MR-data. J Neurosci Methods. (2007) 163:128-36. doi: 10.1016/j.jneumeth.2007.01.026

20. Fox MD, Zhang D, Snyder AZ, Raichle ME. The global signal and observed anticorrelated resting state brain networks. J Neurophysiol. (2009) 101:327083. doi: 10.1152/jn. 90777.2008

21. Power JD, Mitra A, Laumann TO, Snyder AZ, Schlaggar BL, Petersen SE. Methods to detect, characterize, and remove motion artifact in resting state fMRI. Neuroimage. (2014) 84:320-41. doi: 10.1016/j.neuroimage.2013.08.048

22. Smyser CD, Inder TE, Shimony JS, Hill JE, Degnan AJ, Snyder AZ, et al. Longitudinal analysis of neural network development in preterm infants. Cereb Cortex. (2010) 20:2852-62. doi: 10.1093/cercor/bhq035

23. Corbetta M, Kincade JM, Ollinger JM, McAvoy MP, Shulman GL. Voluntary orienting is dissociated from target detection in human posterior parietal cortex. Nat Neurosci. (2000) 3:292-7. doi: 10.1038/73009

24. Yeo BT, Krienen FM, Sepulcre J, Sabuncu MR, Lashkari D, Hollinshead $\mathrm{M}$, et al. The organization of the human cerebral cortex estimated by intrinsic functional connectivity. J Neurophysiol. (2011) 106:1125-65. doi: 10.1152/jn.00338.2011

25. Brier MR, Thomas JB, Snyder AZ, Benzinger TL, Zhang D, Raichle $\mathrm{ME}$, et al. Loss of intranetwork and internetwork resting state functional connections with Alzheimer's disease progression. J Neurosci. (2012) 32:88909. doi: 10.1523/JNEUROSCI.5698-11.2012

26. Chhatwal JP, Schultz AP, Johnson KA, Hedden T, Jaimes S, Benzinger TLS, et al. Preferential degradation of cognitive networks differentiates Alzheimer's disease from ageing. Brain. (2018) 141:1486-500. doi: 10.1093/brain/ awy053

27. Thomas JB, Brier MR, Snyder AZ, Vaida FF, Ances BM. Pathways to neurodegeneration: effects of HIV and aging on resting-state functional connectivity. Neurology. (2013) 80:1186-93. doi: 10.1212/WNL.0b013e318288792b

28. Friederici AD, Gierhan SM. The language network. Curr Opinion Neurobiol. (2013) 23:250-4. doi: 10.1016/j.conb.2012.10.002

29. Wagner S, Sebastian A, Lieb K, Tuscher O, Tadic A. A coordinatebased ALE functional MRI meta-analysis of brain activation during verbal fluency tasks in healthy control subjects. BMC Neurosci. (2014) 15:19. doi: 10.1186/1471-2202-15-19

30. Pernet CR, McAleer P, Latinus M, Gorgolewski KJ, Charest I, Bestelmeyer $\mathrm{PE}$, et al. The human voice areas: spatial organization and inter-individual variability in temporal and extra-temporal cortices. Neuroimage. (2015) 119:164-74. doi: 10.1016/j.neuroimage.2015.06.050

31. Price CJ. A review and synthesis of the first 20 years of PET and fMRI studies of heard speech, spoken language and reading. Neuroimage. (2012) 62:816-47. doi: 10.1016/j.neuroimage.2012.04.062

32. Tremblay P, Dick AS. Broca and Wernicke are dead, or moving past the classic model of language neurobiology. Brain Lang. (2016) 162:60-71. doi: 10.1016/j.bandl.2016.08.004

33. Benjamin CF, Walshaw PD, Hale K, Gaillard WD, Baxter LC, Berl MM, et al. Presurgical language fMRI: Mapping of six critical regions. Hum Brain Mapp. (2017) 38:4239-55. doi: 10.1002/hbm.23661

34. Van Essen DC. A Population-Average, Landmark- and Surface-based (PALS) atlas of human cerebral cortex. Neuroimage. (2005) 28:635-62. doi: 10.1016/j.neuroimage.2005.06.058

35. Dosenbach NU, Fair DA, Miezin FM, Cohen AL, Wenger KK, Dosenbach RA, et al. Distinct brain networks for adaptive and stable task control in humans. Proc Natl Acad Sci USA. (2007) 104:11073-8. doi: 10.1073/pnas.0704320104

36. Ptak R, Schnider A, Fellrath J. The dorsal frontoparietal network: a core system for emulated action. Trends Cogn Sci. (2017) 21:589-99. doi: 10.1016/j.tics.2017.05.002

37. Seeley WW, Menon V, Schatzberg AF, Keller J, Glover GH, Kenna $\mathrm{H}$, et al. Dissociable intrinsic connectivity networks for salience processing and executive control. J Neurosci. (2007) 27:2349-56. doi: 10.1523/JNEUROSCI.5587-06.2007

38. Dosenbach NU, Visscher KM, Palmer ED, Miezin FM, Wenger KK, Kang $\mathrm{HC}$, et al. A core system for the implementation of task sets. Neuron. (2006) 50:799-812. doi: 10.1016/j.neuron.2006.04.031

39. Nelson SM, Dosenbach NU, Cohen AL, Wheeler ME, Schlaggar BL, Petersen SE. Role of the anterior insula in task-level control and focal attention. Brain Struct Funct. (2010) 214:669-80. doi: 10.1007/s00429-010-0260-2

40. Hugdahl K, Raichle ME, Mitra A, Specht K. On the existence of a generalized non-specific task-dependent network. Front Hum Neurosci. (2015) 9:430. doi: 10.3389/fnhum.2015.00430

41. Shackman AJ, Salomons TV, Slagter HA, Fox AS, Winter JJ, Davidson RJ. The integration of negative affect, pain and cognitive control in the cingulate cortex. Nat Rev Neurosci. (2011) 12:154-67. doi: 10.1038/nrn2994

42. Kolling N, Wittmann MK, Behrens TE, Boorman ED, Mars RB, Rushworth MF. Value, search, persistence and model updating in anterior cingulate cortex. Nat Neurosci. (2016) 19:1280-5. doi: 10.1038/nn.4382

43. Neta M, Miezin FM, Nelson SM, Dubis JW, Dosenbach NU, Schlaggar BL, et al. Spatial and temporal characteristics of error-related activity in the human brain. J Neurosci. (2015) 35:253-66. doi: 10.1523/JNEUROSCI.1313-14.2015

44. Carter CS, van Veen V. Anterior cingulate cortex and conflict detection: an update of theory and data. Cogn Affect Behav Neurosci. (2007) 7:367-79. doi: 10.3758/CABN.7.4.367

45. Kelley TA, Serences JT, Giesbrecht B, Yantis S. Cortical mechanisms for shifting and holding visuospatial attention. Cereb Cortex. (2008) 18:114-25. doi: $10.1093 /$ cercor/bhm036

46. Corbetta M, Shulman GL. Spatial neglect and attention networks. Annu Rev Neurosci. (2011) 34:569-99. doi: 10.1146/annurev-neuro-061010-113731

47. Karnath HO. Spatial attention systems in spatial neglect. Neuropsychologia. (2015) 75:61-73. doi: 10.1016/j.neuropsychologia.2015.05.019

48. Spreng RN, Stevens WD, Chamberlain JP, Gilmore AW, Schacter DL. Default network activity, coupled with the frontoparietal control network, supports goal-directed cognition. Neuroimage. (2010) 53:303-17. doi: 10.1016/j.neuroimage.2010.06.016

49. Sadaghiani S, Scheeringa R, Lehongre K, Morillon B, Giraud AL, D’Esposito $\mathrm{M}$, et al. alpha-band phase synchrony is related to activity in the 
fronto-parietal adaptive control network. J Neurosci. (2012) 32:14305-10. doi: 10.1523/JNEUROSCI.1358-12.2012

50. Genovesio A, Wise SP, Passingham RE. Prefrontal-parietal function: from foraging to foresight. Trends Cogn Sci. (2014) 18:72-81. doi: 10.1016/j.tics.2013.11.007

51. Spetzler RF, Martin NA. A proposed grading system for arteriovenous malformations. J Neurosurg. (1986) 65:476-83. doi: 10.3171/jns.1986.65.4.0476

52. Baldassarre A, Ramsey L, Hacker CL, Callejas A, Astafiev SV, Metcalf NV, et al. Large-scale changes in network interactions as a physiological signature of spatial neglect. Brain. (2014) 137(Pt 12):3267-83. doi: 10.1093/brain/awu297

53. Cohen RA, Kaplan RF, Zuffante P, Moser DJ, Jenkins MA, Salloway S, et al. Alteration of intention and self-initiated action associated with bilateral anterior cingulotomy. J Neuropsychiatry Clin Neurosci. (1999) 11:444-53. doi: 10.1176/jnp.11.4.444

54. Rutten GJ, Ramsey NF, van Rijen PC, Noordmans HJ, van Veelen CW. Development of a functional magnetic resonance imaging protocol for intraoperative localization of critical temporoparietal language areas. Ann Neurol. (2002) 51:350-60. doi: 10.1002/ana.10117

55. Zaca D, Jarso S, Pillai JJ. Role of semantic paradigms for optimization of language mapping in clinical FMRI studies. AJNR Am J Neuroradiol. (2013) 34:1966-71. doi: 10.3174/ajnr.A3628

56. Black DF, Vachha B, Mian A, Faro SH, Maheshwari M, Sair HI, et al. American society of functional neuroradiology-recommended fMRI paradigm algorithms for presurgical language assessment. AJNR Am J Neuroradiol. (2017) 38:E65-73. doi: 10.3174/ajnr.A5345
57. Agarwal S, Sair HI, Yahyavi-Firouz-Abadi N, Airan R, Pillai JJ. Neurovascular uncoupling in resting state fMRI demonstrated in patients with primary brain gliomas. J Magn Reson Imaging. (2016) 43:620-6. doi: 10.1002/jmri.25012

58. Mitchell TJ, Hacker CD, Breshears JD, Szrama NP, Sharma M, Bundy DT, et al. A novel data-driven approach to preoperative mapping of functional cortex using resting-state functional magnetic resonance imaging. Neurosurgery. (2013) 73:969-82; discussion 982-963. doi: 10.1227/NEU.00000000000 00141

59. Szaflarski JP, Gloss D, Binder JR, Gaillard WD, Golby AJ, Holland SK, et al. Practice guideline summary: use of fMRI in the presurgical evaluation of patients with epilepsy: report of the guideline development, dissemination, and implementation subcommittee of the american academy of neurology. Neurology. (2017) 88:395-402. doi: 10.1212/WNL.00000000000 03532

Conflict of Interest: The authors declare that the research was conducted in the absence of any commercial or financial relationships that could be construed as a potential conflict of interest.

Copyright (c) 2020 Luckett, Lee, Park, Dierker, Daniel, Seitzman, Hacker, Ances, Leuthardt, Snyder and Shimony. This is an open-access article distributed under the terms of the Creative Commons Attribution License (CC BY). The use, distribution or reproduction in other forums is permitted, provided the original author $(s)$ and the copyright owner(s) are credited and that the original publication in this journal is cited, in accordance with accepted academic practice. No use, distribution or reproduction is permitted which does not comply with these terms. 